\title{
A Global Game with Heterogenous Priors 1
}

\author{
Wolfgang Kuhle \\ Max Planck Institute for Research on Collective Goods, Kurt-Schumacher-Str. 10, 53113 \\ Bonn, Germany. Email: kuhle@coll.mpg.de.
}

\begin{abstract}
This paper relaxes the common prior assumption in the public and private information game of Morris and Shin (2000, 2004). For the generalized game, where the agent's prior expectations are heterogenous, it derives a sharp condition for the emergence of unique/multiple equilibria. This condition indicates that unique equilibria are played if player's public disagreement is substantial. If disagreement is small, equilibrium multiplicity depends on the relative precisions of private signals and subjective priors. Extensions to environments with public signals of exogenous and endogenous quality show that prior heterogeneity, unlike heterogeneity in private information, provides a robust anchor for unique equilibria. Finally, irrespective of whether priors are common or not, we show that public signals can ensure equilibrium uniqueness, rather than multiplicity, if they are sufficiently precise.
\end{abstract}

Keywords: Global Games, Equilibrium Selection, Heterogenous Priors, Thin out Effect

JEL: D53, D82, D83

\section{Introduction}

Heterogeneity in private beliefs is the core component of global coordination games. In the original two-player games introduced by Carlsson and van Damme (1993) and Rubinstein (1989), as well as in the Morris and Shin (1998) extension with a continuum of players, it is a small perturbation away from common knowledge, which selects unique equilibria. This pivotal role of private beliefs was put into perspective by Morris and Shin (2000, 2004), Hellwig (2002), and Metz (2002), who introduce common priors and public signals into the global games model. In such extended settings, it turns out that the global game structure, and its inductive equilib-

\footnotetext{
${ }^{1}$ I thank Martin Hellwig for bringing the literature on global games to my attention. I thank Sophie Bade, Nataliya Demchenko, Alia Gizatulina, Olga Gorelkina, Dominik Grafenhofer, Carl Christian von Weizsï ¿ $\frac{1}{2}$ cker, and seminar participants in Bonn for helpful and encouraging discussions.
} 
rium selection mechanism, is distorted. And it depends on the relative precision of public and private information whether agents can coordinate on multiple equilibria.

Given that the dispersion of private beliefs is pivotal to the global games approach, it is complementary to study how a relaxation of the common prior assumption, which adds a new dimension to the dispersion of private beliefs, affects equilibrium outcomes. To address this question, we study coordination games which are played by a continuum of agents that differ with regard to (i) private information and (ii) the mean of their prior over an unknown fundamental that characterizes the game's payoffs. While the heterogeneity in private signals is unobservable to players, players will know the distribution of priors. That is, we study how agents can coordinate on equilibria in environments, where they know that others hold, and act upon, views that they commonly know to disagree with. The main result shows that if player's public disagreement regarding the coordination game's payoff structure is substantial, then they play unique equilibria. That is, unique equilibria can be selected through the dispersion of priors.

We derive our results in two steps. First, we isolate the pure interaction between private signals and dispersed priors in an environment which abstracts from public signals. Second, we add three different types of public signals to examine the robustness of our results. In the first environment, which abstracts from public signals, we find that heterogeneity in beliefs, which originates from the dispersion of prior expectations $\mu$, contributes to equilibrium uniqueness. Dispersion in private signals, on the contrary, induces equilibrium multiplicity. In the extended settings, where we introduce different types of public signals, we find that prior heterogeneity still unambiguously contributes towards equilibrium uniqueness. The role of private signals, on the contrary, is ambiguous and depends critically on the specification of the public signal. The main finding in the second part of the paper is therefore that heterogeneity in priors, unlike heterogeneity in private information, provides a robust anchor for unique equilibria. Put differently, we show that heterogeneous priors robustly select unique equilibria in rather diverse environments. In turn, we compare this finding to the literature where the presence of common priors induces multiple rather than unique equilibria.

Finally, as a byproduct of our analysis, we find that public signals in themselves, irrespective of whether priors are heterogenous or common, have an ambiguous effect on equilibrium multiplicity: increases in the public signal's precision can ensure equilibrium uniqueness. This result 
is of interest in comparison with those of Morris and Shin (2000, 2004), Hellwig (2002), Metz (2002), Angeletos and Werning (2006) who find that increases in the public signal's precision unambiguously induce multiple rather than unique equilibria.2

Compared to the literature, we extend the canonic common prior coordination games by Morris and Shin $(2000,2004)$ to a setting where agents' prior distribution's mean $\mu$, regarding the true state of the world, are normally distributed over the economy's population. In the model of Morris and Shin (2000, 2004), equilibrium uniqueness is ensured iff $\frac{\sqrt{\alpha_{x}}}{\alpha_{p}} \geq \frac{1}{\sqrt{2 \pi}}$; where $\alpha_{x}$ is the private signal's precision and $\alpha_{p}$ is the prior's precision. For the generalized game, where subjective prior expectations $\mu$ are distributed with variance $\sigma_{\mu}$, we obtain $\sqrt{\left(\frac{\sqrt{\alpha_{x}}}{\alpha_{p}}\right)^{2}+\sigma_{\mu}^{2}} \geq \frac{1}{\sqrt{2 \pi}}$ as an analog condition for equilibrium uniqueness. This formula's main strength is that it isolates how prior heterogeneity $\sigma_{\mu}$ affects the number of possible equilibria. In general, the condition shows that incomplete information games where the population of players is polarized such that there are two large tails have unique equilibria. Or, equivalently, unique equilibria are ensured if there is only a small group of agents which have "intermediate" or "moderate" beliefs. Put differently, increases in the priors dispersion, $\sigma_{\mu}$, "thin out" the group of agents with "moderate" beliefs which can, potentially, coordinate on multiple equilibria. Technically, equilibrium multiplicity depends on the relative precisions of private information $\left(\alpha_{x}\right)$ and the subjective prior $\left(\alpha_{p}\right)$ if the prior's dispersion is small $\left(\sigma_{\mu}<\frac{1}{\sqrt{2 \pi}}\right)$. For sufficiently dispersed priors, $\left(\sigma_{\mu}>\frac{1}{\sqrt{2 \pi}}\right)$, equilibria are unique, irrespective of the relative precisions of private signals and priors. The original uniqueness condition for the common prior economy $\left(\frac{\sqrt{\alpha_{x}}}{\alpha_{p}} \geq \frac{1}{\sqrt{2 \pi}}\right)$ obtains as prior dispersion $\left(\sigma_{\mu}\right)$ vanishes.

In Section 3, we extend the information structure and embed our coordination game into three different public signal environments. These extensions provide a background to study the fundamental difference between heterogeneity in priors and private signals. The main finding in this section is that only heterogeneity in priors selects unique equilibria reliably. On the contrary, the role of both private and public signals changes from environment to environment. These findings originate, first of all, from an environment where the public signal reveals the true state of the game with exogenous quality; secondly, from an environment that may be seen as a financial markets context, where stock prices aggregate and reveal dispersed private

\footnotetext{
${ }^{2}$ There will be two classes of equilibria in this setting, and the public signal's precision ensures uniqueness for every given signal realization. However, if the public signal's precision is sufficiently high, there may be multiplicity in the public signal's realization itself.
} 
information with endogenous quality; and thirdly, from an environment with public signals that allow agents to observe each other's actions. Comparison of all three public signal settings shows that the prior's dispersion $\sigma_{\mu}$ is the only parameter which can ensure unique equilibria in all environments. The implications of the public and private signals' precisions, however, vary from case to case. In particular, increases in the private signal's precision ensure uniqueness (multiplicity) when public signals are of exogenous (endogenous) precision.

Recently, Steiner and Stewart (2008), Izmalkov and Yildiz (2010), and Mathevet (2012) have introduced heterogenous priors into global games. Steiner and Stewart (2008), Izmalkov and Yildiz (2010), and Mathevet (2012) focus, respectively, on learning, rationalizability of strategies, and applications to mechanism design. Unlike Steiner and Stewart (2008) and Mathevet (2012), who focus on N-player games, we study games with a continuum of players as in Izmalkov and Yildiz (2010). Contrary to Izmalkov and Yildiz (2010), who focus on the private information limit 3 of Carlsson and van Damme (1993), which carries over to these more general games, the present paper develops a sharp characterization of equilibrium multiplicity at and away from the private information limit 4 The present paper is therefore the first to derive closed form expressions for the emergence of multiple equilibria in large games with heterogeneous priors. Moreover, by means of the public signal environments, it provides a framework to interpret the role of prior heterogeneity in the global games structure 5

Regarding the heterogeneity in priors, we note that Morris and Shin (1998, 2000, 2004) embed their common prior incomplete information coordination games in a currency crises,

\footnotetext{
${ }^{3}$ In the "private information limit", the private signal's precision goes to infinity. A crucial consequence of this is that the importance of priors for the agent's decisions vanishes.

${ }^{4}$ Izmalkov and Yildiz (2010) discuss prior heterogeneity in a game with a continuum of players. Izmalkov and Yildiz (2010), p. 25, focus on the formation of individual threshold strategies and avoid the "delicate" question of equilibrium multiplicity, which is the focus of the present paper. More precisely, Izmalkov and Yildiz (2010) study games with heterogenous priors ("sentiments") and characterize for a family of coordination games how prior dispersion affects the unique threshold equilibrium which is ensured once private information becomes sufficiently precise. Due to the focus on the private information limit, it remains an open question how prior heterogeneity affects the number of equilibria in those cases where private signals are not arbitrarily precise. This question is the focus of the current paper. Finally, while Izmalkov and Yildiz (2010) characterize games with general distribution functions, the present characterization of equilibrium uniqueness and multiplicity, away from the limit where private information is very precise, relies on the afore mentioned normality assumptions.

5 Steiner and Stewart (2008), Izmalkov and Yildiz (2010), and Mathevet (2012) abstract from public signals.
} 
bank-run and debt-run context respectively. In these contexts, our assumption of a prior distribution $\mu_{i} \sim \mathcal{N}\left(E[\mu], \sigma_{\mu}^{2}\right)$, which is known to all agents, may be interpreted as a publicly observable distribution of exchange rate forecasts or credit ratings. Such a distribution of conflicting beliefs, may also be seen as consistent with publicly disclosed long and short positions that large investors take in a firm's stock or debt. While Morris (1995) and Sethi and Yildiz (2012), discuss heterogenous priors and their origins in detail, we will take prior heterogeneity as a given. In turn, we focus purely on the implications that public disagreement has for the emergence of multiple equilibria in the global games framework.

The rest of the paper is organized as follows. In Section 2.1, we introduce the model. Section 2.2 contains the main result. In Section 3 , we reflect on our findings in three distinct public signal environments. Section 4 concludes.

\section{The Model}

To isolate the implications of prior dispersion, we start with a setting that differs only with regard to the heterogeneity of priors from the canonic framework introduced by Morris and Shin (2000, 2004).

\subsection{Strategies, Payoffs and Information}

There is a status quo and a unit measure of agents indexed by $i \in[0,1]$. Each of these agents $i$ can choose between two actions $a_{i} \in\{0,1\}$. Choosing $a_{i}=1$ means to attack the status quo. Choosing $a_{i}=0$ means that the agent does not attack the status quo. An attack on the status quo is associated with a cost $c \in(0,1)$. If the attack is successful, the status quo is abandoned and attacking agents receive a net payoff $1-c>0$. If the attack is not successful, an attacking agent's net payoff is $-c$. The payoff for an agent who does not attack is normalized to zero.

The status quo is abandoned if the aggregate size of the attack $A:=\int_{0}^{1} a_{i} d i$ exceeds the strength of the status quo $\theta$, i.e., if $A>\theta$. Otherwise, if $A<\theta$, the status quo is maintained and the attack fails. Regarding the fundamental, $\theta$, each agent $i$ holds a prior belief $\theta \sim$ $\mathcal{N}\left(\mu_{i}, \sigma_{p}^{2}\right)$. In our setting, we assume that the priors, regarding the fundamental $\theta$, are normally distributed across the population, i.e., $\mu_{i} \sim \mathcal{N}\left(E[\mu], \sigma_{\mu}^{2}\right)$. And the distribution of priors is common knowledge. In addition to the prior, each agent receives a private signal $x_{i}=\theta+\sigma_{x} \xi_{i}$. 
Where signal noise $\xi_{i} \sim \mathcal{N}(0,1)$ is i.i.d. across the population. We parameterize the information structure in terms of precisions $\alpha_{x} \equiv \frac{1}{\sigma_{x}^{2}}$ and $\alpha_{p} \equiv \frac{1}{\sigma_{p}^{2}}$. Agents use their information to calculate expected utility

$$
E\left[U\left(A, \theta, c, a_{i}\right) \mid \mu_{i}, x_{i}\right]=a_{i}\left(1 \times P\left(\theta<A \mid \mu_{i}, x_{i}\right)-c\right)
$$

Where $P\left(\theta<A \mid \mu_{i}, x_{i}\right)$ is the probability that the attack is successful given signal $x_{i}$ and prior belief $\mu_{i}$. Regarding payoffs, (1) reflects that an agent $i$, who does not attack $\left(a_{i}=0\right)$ receives a safe payoff of 0 , while an agent who does attack $\left(a_{i}=1\right)$ receives $1-c$ if the attack is successful, and $-c$ otherwise. To close the description of agents' information, we note that the distributions of $x_{i}$ and $\mu_{i}$ are commonly known to all players. With the private choice problem in place, we now characterize equilibrium.

\subsection{Equilibrium}

As Morris and Shin (1998, 2004), we focus on monotone threshold equilibria. Each threshold equilibrium will be characterized by a pair $\psi^{*}, \theta^{*}$. Where $\theta^{*}$ separates values $\theta<\theta^{*}$ for which the status quo is abandoned from values $\theta>\theta^{*}$ where it prevails. The threshold level $\psi^{*} \equiv \frac{\alpha_{x}}{\alpha} x^{*}+\frac{\alpha_{p}}{\alpha} \mu^{*}$ summarizes the critical pairs $x^{*}, \mu^{*}$, for which agents are just indifferent between attacking and not attacking 6 The equilibrium pairs, $\psi^{*}, \theta^{*}$, are determined by the simultaneous evaluation of the payoff indifference condition and the critical mass condition. The payoff indifference condition, PIC, follows directly from the individual choice problem (1). Taking the threshold level $\theta^{*}$ as given, we have 18

$$
P\left(\theta \leq \theta^{*} \mid x^{*}, \mu^{*}\right)=c \quad \Leftrightarrow \quad \Phi\left(\sqrt{\alpha}\left(\theta^{*}-\frac{\alpha_{x}}{\alpha} x^{*}-\frac{\alpha_{p}}{\alpha} \mu^{*}\right)\right)=c ; \quad \alpha=\alpha_{x}+\alpha_{p},
$$

where $\Phi()$ is the cumulative of the standard normal distribution. The PIC (2) characterizes those pairs $x^{*}, \mu^{*}$ which are such that the agent is indifferent between attacking and not at-

\footnotetext{
${ }^{6}$ In the context of the currency crises model of Morris and Shin (1998), attacking agents would sell short a country's currency, and the central bank's reserves $\theta$ are either sufficient $(\theta>A)$ to defend the peg or not $(\theta<A)$. In another interpretation, agents can run on/sell short a firm's debt, and if the firm's financial strength is insufficient it defaults.

${ }^{7}$ See Raiffa and Schlaifer (2000), p. 250, for the standard results on prior and posterior distributions of normally distributed variables which are used throughout the paper.

${ }^{8}$ Note that we already use the (forthcoming) critical mass condition, (44), which requires that $\theta^{*} \equiv A\left(\psi^{*}, \theta^{*}\right)$, and replace $A$ with $\theta^{*}$ in (1) to obtain (2).
} 
tacking. To work with Condition (2), we define $\psi \equiv \frac{\alpha_{x}}{\alpha} x+\frac{\alpha_{p}}{\alpha} \mu$ such that (2) writes:

$$
P\left(\theta \leq \theta^{*} \mid \psi^{*}\right)=c \quad \Leftrightarrow \quad \Phi\left(\sqrt{\alpha}\left(\theta^{*}-\psi^{*}\right)\right)=c ; \quad \alpha=\alpha_{x}+\alpha_{p}
$$

And agents who receive $\psi \leq \psi^{*}$, which is evidence of a weak fundamental, attack. Agents who receive $\psi>\psi^{*}$ do not attack since they believe in a strong fundamental, which makes a successful attack unlikely.

The critical mass condition, CMC, takes the cutoff value $\psi^{*}$ as given and determines the threshold $\theta^{*}$, where the attack is just strong enough to overwhelm the status quo. To calculate the mass of attacking agents, we note that $\psi \mid \theta \sim \mathcal{N}\left(\frac{\alpha_{x}}{\alpha} \theta+\frac{\alpha_{p}}{\alpha} E[\mu], \alpha_{\psi}^{-1}\right)$ where $\alpha_{\psi} \equiv \frac{\alpha^{2}}{\alpha_{x}+\alpha_{p}^{2} \sigma_{\mu}^{2}}$ and $\alpha=\alpha_{x}+\alpha_{p}$. The CMC therefore writes:

$$
A\left(\psi^{*}, \theta^{*}\right) \equiv P\left(\psi<\psi^{*} \mid \theta^{*}\right)=\theta^{*} \quad \Leftrightarrow \quad \Phi\left(\sqrt{\alpha_{\psi}}\left(\psi^{*}-\frac{\alpha_{x}}{\alpha} \theta^{*}-\frac{\alpha_{p}}{\alpha} E[\mu]\right)\right)=\theta^{*} .
$$

Again, $\Phi()$ is the cumulative of the standard normal distribution. Regarding (4), we note that it implies that there exists only one $\psi^{*}$ for every $\theta^{*}$. Simultaneous evaluation of (3) and (4) yields threshold equilibria $\psi^{*}, \theta^{*}$ :

Proposition 1. The equilibrium $\psi^{*}, \theta^{*}$ is unique, for all parameter pairs, if and only if $\sqrt{\left(\frac{\sqrt{\alpha_{x}}}{\alpha_{p}}\right)^{2}+\sigma_{\mu}^{2}} \geq \frac{1}{\sqrt{2 \pi}}$.

Proof. We solve (3) for the threshold level $\psi^{*}=-\Phi^{-1}(c) \frac{1}{\sqrt{\alpha}}+\theta^{*}$, and substitute $\psi^{*}$ into (4) to obtain a one-dimensional equation in $\theta^{*}$ :

$$
\Phi\left(\sqrt{\alpha_{\psi}}\left(-\Phi^{-1}(c) \frac{1}{\sqrt{\alpha}}+\theta^{*}-\frac{\alpha_{x}}{\alpha} \theta^{*}-\frac{\alpha_{p}}{\alpha} E[\mu]\right)\right)=\theta^{*} .
$$

The sufficient condition for uniqueness of the threshold $\theta^{*}$ is therefore:

$$
\begin{aligned}
& \sqrt{\alpha_{\psi}} \frac{\alpha_{p}}{\alpha} \phi\left(\sqrt{\alpha_{\psi}}\left(-\Phi^{-1}(c) \frac{1}{\sqrt{\alpha}}+\frac{\alpha_{p}}{\alpha} \theta^{*}-\frac{\alpha_{p}}{\alpha} E[\mu]\right)\right) \leqq 1 \\
& \Leftrightarrow \sqrt{\alpha_{\psi}} \frac{\alpha_{p}}{\alpha} \frac{1}{\sqrt{2 \pi}} e^{-\left(\sqrt{\alpha_{\psi}}\left(-\Phi^{-1}(c) \frac{1}{\sqrt{\alpha}}+\frac{\alpha_{p}}{\alpha} \theta^{*}-\frac{\alpha_{p}}{\alpha} E[\mu]\right)\right)^{2} \frac{1}{2}} \leqq 1 .
\end{aligned}
$$

Finally, we recall that $\alpha_{\psi}=\frac{\alpha^{2}}{\sqrt{\alpha_{x}+\alpha_{p}^{2} \sigma_{\mu}^{2}}}$ and take logarithms to obtain:

$$
\ln \left(\frac{1}{\sqrt{2 \pi}} \frac{1}{\sqrt{\left(\frac{\sqrt{\alpha_{x}}}{\alpha_{p}}\right)^{2}+\sigma_{\mu}^{2}}}\right) \leqq\left(\sqrt{\alpha_{\psi}}\left(-\Phi^{-1}(c) \frac{1}{\sqrt{\alpha}}+\frac{\alpha_{p}}{\alpha} \theta^{*}-\frac{\alpha_{p}}{\alpha} E[\mu]\right)\right)^{2} \frac{1}{2}
$$


Accordingly, unique equilibria are ensured once 9

$$
\ln \left(\frac{1}{\sqrt{2 \pi}} \frac{1}{\sqrt{\left(\frac{\sqrt{\alpha_{x}}}{\alpha_{p}}\right)^{2}+\sigma_{\mu}^{2}}}\right) \leq 0 \quad \Leftrightarrow \quad \sqrt{\left(\frac{\sqrt{\alpha_{x}}}{\alpha_{p}}\right)^{2}+\sigma_{\mu}^{2}} \geq \frac{1}{\sqrt{2 \pi}}
$$

In one interpretation, the role of the prior's dispersion $\sigma_{\mu}$ in Condition (7) indicates that polarized economies, with two large groups (tails) which believe that the status quo is going to be maintained (abandoned), have unique equilibria. Put differently, increases in the prior's dispersion $\sigma_{\mu}$ "thin out" the group of agents, around the mean $E[\mu]$ who hold "moderate" beliefs. And it is this group which can potentially coordinate on multiple equilibria. Regarding the prior's weight, $\alpha_{p}$, we find that, for every given prior expectation $\mu$, increases in $\alpha_{p}$ make actions more predictable. This allows agents to coordinate, as in the common prior economy of Morris and Shin (2004), which contributes towards equilibrium multiplicity. Comparison of the two origins of belief heterogeneity indicates that increased dispersion of private signals contributes towards equilibrium multiplicity, while increases in the dispersion of prior beliefs $\mu$ contributes towards uniqueness. In the next section, we show that the thinning-out effect is robust to various changes in the informational environment. And it is the only avenue that can ensure unique equilibria in all three public signal environments that follow.

From a technical perspective, we note that Proposition 1 has two corollaries:

Corollary 1. If the prior $\mu$ is sufficiently dispersed, such that $\sigma_{\mu}>\frac{1}{\sqrt{2 \pi}}$, equilibria are unique irrespective of the relative precision $\frac{\sqrt{\alpha_{x}}}{\alpha_{p}}$ of private signal and prior. And in the case where the private signal is uninformative, $\alpha_{x}=0$, unique (multiple) equilibria exist if $\sigma_{\mu}>(<) \frac{1}{\sqrt{2 \pi}}$.

Corollary 2. The uniqueness condition $\sqrt{\left(\frac{\sqrt{\alpha_{x}}}{\alpha_{p}}\right)^{2}+\sigma_{\mu}^{2}} \geq \frac{1}{\sqrt{2 \pi}}$ converges smoothly to the uniqueness condition, $\frac{\sqrt{\alpha_{x}}}{\alpha_{p}} \geq \frac{1}{\sqrt{2 \pi}}$, of the Morris and Shin (2004) common prior game, as $\sigma_{\mu} \rightarrow 0$.

\section{Public Signals}

To reflect on the role of prior heterogeneity, we introduce three different types of public signals into our baseline setting. Each of these public signals is chosen to isolate particular differences

\footnotetext{
${ }^{9}$ That is, if (7) holds, then (6) never holds with equality for real-valued $\theta^{*}$ 's. Put differently, the polynomial which characterizes those values $\theta^{*}$, for which (6) holds with equality, has two complex roots.
} 
between heterogeneity in priors and heterogeneity in private signals. The main finding in this section is that only heterogeneity in priors selects unique equilibria reliably. That is, if player's disagreement, as measured by $\sigma_{\mu}$, is substantial, then they play unique equilibria irrespective of the particular public signal context. That is, the result from the previous section, i.e., that prior heterogeneity induces equilibrium uniqueness through the thinning-out effect, is robust to the introduction of public signals. On the contrary, the role the private signal will change from environment to environment.

The first public signal, which we introduce into our baseline model from Section 2, is of exogenous quality. That is, it reveals the true fundamental of the game with exogenous precision $\alpha_{z}$. In such an extended setting, we find that the comparative statics of the subjective prior's precision $\alpha_{p}$ change: contrary to the baseline setting, where increases in the prior weight always contribute towards multiplicity, we find that increases in the prior weight can now shift the modified economy from multiplicity towards uniqueness. In a second step, we endogenize the quality of the public signal. To do so, we embed our coordination game into a financial markets context, where a stock price aggregates and reveals dispersed private information. In this setting, we find that the role of private information, with respect to equilibrium multiplicity, is reversed. Namely, equilibrium multiplicity is ensured in the limit where private information becomes arbitrarily precise. On the contrary, the role of prior dispersion is robust to such changes in the model structure.

In the last Section 3.1, we introduce a public signal which partially reveals the aggregate attack $A$. This signal provides an environment where changes in the prior's dispersion may, for intermediate values, induce multiplicity rather than uniqueness. However, in the limit where prior dispersion grows large, it still ensures unique equilibria. Finally, as a byproduct of our analysis in Section 3.1, we find that public signals in themselves have an ambiguous effect on equilibrium multiplicity: sufficiently precise public signals can ensure unique threshold equilibria. This finding is of independent interest in comparison with the games of Morris and Shin (2000, 2004), Hellwig (2002), Metz (2002), Angeletos and Werning (2006), where increases in the public signal's precision unambiguously induce multiple rather than unique threshold equilibria. 
1) Public Signal with Exogenous Precision The public signal

$$
Z=\theta+\sigma_{z} \varepsilon, \quad \varepsilon \sim \mathcal{N}(0,1)
$$

allows agents to forecast the true state of the fundamental with precision $\alpha_{z}=\frac{1}{\sigma_{z}^{2}}$. Agents can therefore use $Z$, in addition to $x$ and $\mu$, to calculate the probability with which the aggregate attack overwhelms the status quo. In Appendix A, we show that, if this signal is used as an additional source of information in the coordination game of Section 2, we have:

Proposition 2. The equilibrium in the public and private information game with heterogenous priors is unique if

$$
\sqrt{\left(\frac{\sqrt{\alpha_{x}}}{\alpha_{p}+\alpha_{z}}\right)^{2}+\sigma_{\mu}^{2} \frac{1}{\left(1+\frac{\alpha_{z}}{\alpha_{p}}\right)^{2}}} \geq \frac{1}{\sqrt{2 \pi}}
$$

In particular, if the prior's dispersion is large, such that $\sigma_{\mu} \frac{1}{1+\frac{\alpha z}{\alpha_{p}}}>\frac{1}{\sqrt{2 \pi}}$, the equilibrium is unique independently of the private signal's precision $\alpha_{x}$.

Compared to the uniqueness condition (17) from the baseline model, we find once again that the modified condition (9) has two elements. The first, $\frac{\sqrt{\alpha_{x}}}{\alpha_{p}+\alpha_{z}}$, reflects the trade-off between private information $\alpha_{x}$ and prior $\alpha_{p}$, described by Morris and Shin (2000, 2004) in an economy without public signals, or respectively, the trade-off between private information and public signals $\alpha_{z}$ which was emphasized by Metz (2002) and Hellwig (2002) in an economy with a uniform uninformative prior. Regarding this first term, we find that public information and prior are perfect substitutes, and both contribute to equilibrium multiplicity. The second term $\sigma_{\mu}^{2} \frac{1}{\left(1+\frac{\alpha_{z}}{\alpha_{p}}\right)^{2}}$, however, shows that increases in the public signal's precision $\alpha_{z}$ reduce the effect of the prior's dispersion, while the prior weight $\alpha_{p}$ increases it. Condition (9) therefore shows that the public signal's precision unambiguously contributes towards multiplicity. Increases in the prior's weight $\alpha_{p}$ on the contrary have an ambiguous consequences as they shift the economy towards uniqueness (multiplicity) if $\sigma_{\mu}^{2}>(<) \frac{\alpha_{x}}{\alpha_{p} \alpha_{z}}$. Finally, (9) reflects that equilibria are unique in the private information limit where $\alpha_{x} \rightarrow \infty$.

2) Public Signal with Endogenous Precision To highlight the different implications of prior dispersion and the dispersion of private signals, we discuss an environment where the global game is embedded in a financial market setting. Following, Atkeson (2000), Angeletos and Wernin (2006), and Hellwig et al. (2006), we introduce a financial market which aggregates dispersed 
private information on the unknown fundamental $\theta$, through its publicly observable stock price, as in Grossman and Stiglitz (1976, 1980), and Hellwig (1980) 10 In one interpretation, the extended model may describe a situation, where bond investors use a firm's stock price to infer its default probability, which is of importance for a coordination game that concerns a potential run on the firm's debt. We show in Appendix B that it is possible to specify the financial market such that the public stock price signal, $Z$, partially reveals the true fundamental $\theta$ :

$$
Z=\theta-\gamma \sigma_{\varepsilon} \sigma_{x}^{2} \varepsilon, \quad \varepsilon \sim \mathcal{N}(0,1)
$$

Thus, the signal's precision $\alpha_{z}=\frac{1}{\left(\gamma \sigma_{\varepsilon}\right)^{2}} \alpha_{x}^{2}$ is an increasing function of the private signal's precision $\alpha_{x}=\frac{1}{\sigma_{x}^{2}}$. That is, the stock price's informativeness increases once the stock investors' information becomes more informative. In the current context, it is important that the precision with which this financial market publicly reveals the true state of the world $\theta$ is increasing faster (in the private signal's precision) than the private signal's precision $\alpha_{x}$ itself. To perform the equilibrium analysis which concerns the coordination game, we recall (9) and note that $\alpha_{z}=\alpha_{z}\left(\alpha_{x}\right)$. This yields

Proposition 3. The equilibrium is unique if

$$
\sqrt{\left(\frac{\sqrt{\alpha_{x}}}{\alpha_{p}+\alpha_{z}\left(\alpha_{x}\right)}\right)^{2}+\sigma_{\mu}^{2} \frac{1}{\left(1+\frac{\alpha_{z}\left(\alpha_{x}\right)}{\alpha_{p}}\right)^{2}}} \geq \frac{1}{\sqrt{2 \pi}}, \quad \alpha_{z}:=\frac{1}{\left(\gamma \sigma_{\varepsilon}\right)^{2}} \alpha_{x}^{2} .
$$

Multiple equilibria exist in the private information limit where $\alpha_{x} \rightarrow \infty$. The equilibrium is unique in the limit where $\sigma_{\mu} \rightarrow \infty$.

Proof. Follows from (11) with $\alpha_{z}\left(\alpha_{x}\right)=\frac{1}{\left(\gamma \sigma_{\varepsilon}\right)^{2}} \alpha_{x}^{2}$.

Proposition 3 establishes that the finding of Angeletos and Werning (2006) carries over to an economy with heterogenous priors. Namely, if stock prices aggregate private information rapidly as in (10), then it is precise private information which ensures equilibrium multiplicity. Moreover, as $\alpha_{z}\left(\alpha_{x}\right)$ becomes large, it marginalizes the influence of prior heterogeneity

\footnotetext{
${ }^{10}$ That is, we assume that agents trade stocks prior to the coordination game. These stocks are traded at a market price $P$ and pay an unknown amount $\theta$. This market price will, in equilibrium, aggregate dispersed private information and reveal the true fundamental $\theta$ partially. Where the partial revelation is due to aggregate noise-trader activity, $\sigma_{\varepsilon} \varepsilon, \varepsilon \sim \mathcal{N}(0,1)$, on the asset's supply side.
} 
$\sigma_{\mu}^{2} \frac{1}{\left(1+\frac{\alpha z}{\alpha_{p}}\right)^{2}}$. Finally, if private noise becomes large as $\alpha_{x} \rightarrow 0$ and thus $\alpha_{z}\left(\alpha_{x}\right) \rightarrow 0$, equilibrium multiplicity depends on prior dispersion, $\sigma_{\mu}$, alone 11

Concerning the different implications of heterogenous priors and heterogenous private signals, the key insight is that the endogeneity of public information inverts the original findings of Morris and Shin (2000, 2004), Metz (2002), and Hellwig (2002), where increases in private information induce equilibrium uniqueness as in (9), where the public signal's precision is exogenous. The same is not true for the role of prior dispersion, which is, contrary to the private signal's dispersion, robust to the introduction of an endogenous public price signal and unambiguously contributes towards equilibrium uniqueness. That is, heterogeneity in priors, unlike heterogeneity in private information, provides a robust anchor for unique equilibria.

\subsection{Observing Other's Actions}

In this section, agents can observe the size of the aggregate attack through a noisy public signal $S=\Phi^{-1}(A)+\sigma_{\varepsilon} \varepsilon$ where $\varepsilon \sim \mathcal{N}(0,1)$. While games where agents can observe each other's actions were already studied by Minelli and Polemarchakis (2003), the current signal specification is taken from Dasgupta (2007) and Angeletos and Werning (2006), since it allows to preserve normal distributions. The following discussion of equilibrium uniqueness is accordingly parallel to that in Angeletos and Werning (2006); and their results obtain as special cases where priors are uninformative, i.e., where $\alpha_{p}=0$. Compared to the analysis in Angeletos and Werning (2006) we note that public signals of high precision can ensure unique threshold equilibria in our specification if priors are informative. That is, the role of the public signal in Angeletos and Werning (2006), pp. 1733-1734, depends critically on the absence of an informative prior 12

\footnotetext{
${ }^{11}$ This finding naturally differs from Angeletos and Werning $(2006)$, where $\sigma_{\mu}=0$, such that noisy private signals unambiguously induce unique equilibria when public information is endogenous once $\alpha_{x} \rightarrow 0$ and $\alpha_{z}\left(\alpha_{x}\right) \rightarrow 0$. Related to this observation, we note that among all parameters, $\alpha_{x}, \alpha_{p}, \sigma_{\mu}$, the prior's dispersion $\sigma_{\mu}$ is the only parameter which can ensure equilibrium uniqueness regardless of the values of the remaining parameters.

${ }^{12}$ More precisely, for a priorless game, Angeletos and Werning (2006) show that threshold equilibria are always unique, but there may exist multiple equilibria in "strategies". In the present model, which includes informative (possibly unique) priors, we show that multiple threshold equilibria may exist. However, if the public signal, over others' actions $A$, is sufficiently precise then threshold equilibria are always unique. The observation that public signals of high quality can ensure unique rather than multiple threshold equilibria is of interest
} 
Compared to the previous two signal environments, signal $S$ carries two types of information. First, similar to signals (8) and (10), the signal $S$ allows agents to make inference on the true fundamental $\theta$ since $A=A\left(\theta, \psi^{*}\right)$. Second, unlike signals (8) and (10), the particular signal realization $S$ is endogenous in the sense that $S$ is implicitly defined by $S=\Phi^{-1}\left(A\left(\theta, \psi^{*}(S)\right)\right)+$ $\sigma_{\varepsilon} \varepsilon$. And there may exist multiple signal values $S$ for every given pair $\theta, \varepsilon$. We examine these potential sources of multiplicity, namely equilibrium multiplicity in thresholds $\theta^{*}, \psi^{*}$ and equilibrium multiplicity in strategies $S, \psi^{*}(S)$ in separate steps.

3) Equilibrium Multiplicity in Thresholds In the augmented game, with heterogeneous priors, where agents observe the aggregate attack through signal $S$, we have:

$$
\begin{aligned}
& S=\Phi^{-1}(A)+\sigma_{\varepsilon} \varepsilon, \quad \varepsilon \sim \mathcal{N}(0,1) \\
& A \equiv P\left(\psi \leq \psi^{*}(S) \mid \theta^{*}\right)=\theta^{*} \\
& P\left(\theta \leq \theta^{*} \mid \psi^{*}, S\right)=c .
\end{aligned}
$$

Evaluation of (12)-(14) yields:

Proposition 4. For every given signal realization $S$, threshold equilibria $\theta^{*}, \psi^{*}$ are unique if $\frac{1}{\sqrt{2 \pi}} \leq \frac{\left(\alpha_{x}+\alpha_{z}\right)}{\alpha_{x}} \sqrt{\left(\frac{\sqrt{\alpha_{x}}}{\alpha_{p}}\right)^{2}+\sigma_{\mu}^{2}}$ where $\alpha_{z}=\frac{\alpha_{x}^{2} \alpha_{\psi}}{\sigma_{\varepsilon}^{2} \alpha^{2}}=\frac{\alpha_{x}^{2}}{\sigma_{\varepsilon}^{2}\left(\alpha_{x}+\alpha_{p}^{2} \sigma_{\mu}^{2}\right)}$. And equilibria are unique when priors are either sufficiently dispersed or when the public signal is sufficiently precise.

Proof. See Appendix C

With regard to the role of prior dispersion, Proposition 4 shows that prior dispersion contributes towards equilibrium uniqueness in the generalized setting where agents can observe each other's actions 13 The more significant finding, however, is that the public signal's precision induces equilibrium uniqueness rather than multiplicity. That is, in the present framework, we find that the public signal allows agents to coordinate on one particular equilibrium rather than multiple equilibria as in Morris and Shin (2000, 2004), Metz (2002), Hellwig (2002), and Angeletos and Werning (2006). Moreover, the comparative statics with regard to the public in comparison with Morris and Shin (2000, 2004), Metz (2002), and Hellwig (2002), who show that multiple threshold equilibria emerge once public signals are of high quality.

${ }^{13}$ Note that increases in the prior's dispersion reduce the public signal's precision $\alpha_{z}=\frac{\alpha_{x}^{2} \alpha_{\psi}}{\sigma_{\varepsilon}^{2} \alpha^{2}}=\frac{\alpha_{x}^{2}}{\sigma_{\varepsilon}^{2}\left(\alpha_{x}+\alpha_{p}^{2} \sigma_{\mu}^{2}\right)}$; for intermediate values of $\sigma_{\mu}$, it is therefore not necessarily true that increases in $\sigma_{\mu}$ contribute towards uniqueness. 
signal carry over to the unique prior economy where $\sigma_{\mu}=0$. Finally, for an uninformative prior where $\alpha_{p}=0$, we find that the uniqueness result of Angeletos and Werning (2006) obtains as a special case 14

4) Equilibrium Multiplicity in Strategies In this paragraph, we study the uniqueness of the equilibrium with respect to the signal $S$. In Appendix $\mathrm{C}$, we show that signal $S$ is, in equilibrium, equivalent to a signal $Z(S)=\frac{\alpha}{\alpha_{x}} \psi^{*}(S)-\frac{\alpha}{\alpha_{x}} \frac{1}{\sqrt{\alpha_{\psi}}} S=\theta+\frac{\alpha_{p}}{\alpha_{x}} E[\mu]-\sigma_{\varepsilon} \frac{\alpha}{\alpha_{x}} \frac{1}{\sqrt{\alpha_{\psi}}} \varepsilon$. And multiple equilibria can emerge in the sense 15 that there may exist several signal values $S$, and thus several values $\psi^{*}(S)$, which satisfy $Z(S)=\bar{Z}$. Concerning this potential source of equilibrium multiplicity we note

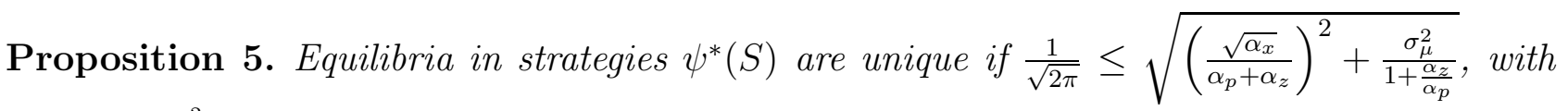
$\alpha_{z}=\frac{\alpha_{x}^{2}}{\sigma_{\varepsilon}^{2}\left(\alpha_{x}+\alpha_{p}^{2} \sigma_{\mu}^{2}\right)}$. In the limit, where $\sigma_{\mu} \rightarrow \infty$ there exists a unique equilibrium. In the limit where $\alpha_{z} \rightarrow \infty$, there exist multiple equilibria in strategies.

Proof. See Appendix C

Comparison of propositions 4 and 5 with regard to the prior's dispersion yields an important corollary:

Corollary 3. The overall equilibrium is unique in the limit where $\sigma_{\mu} \rightarrow \infty$.

Corollary 3 underscores the main result of the paper, i.e., it confirms that sufficiently dispersed priors ensure unique equilibria. The public signal's precision has a more differentiated influence on equilibria: it ensures uniqueness in thresholds if it is sufficiently precise, but at the same time it opens the door to multiple equilibria in strategies.

\footnotetext{
${ }^{14}$ In a setting with a uniform uninformative prior, Angeletos and Werning (2006), pp. 1733-1734, prove that threshold equilibria are always unique irrespective of the precisions $\alpha_{z}$ and $\alpha_{x}$. To obtain this result, one can either repeat the calculations in Appendix $\mathrm{C}$ with $\alpha_{p}=0$. Alternatively, one can observe that the uniqueness condition in Proposition 4 is always satisfied once a sufficiently small value $\alpha_{p}$ is chosen.

${ }^{15}$ At this point we do not discuss the conceptual validity of this alternative type/source of equilibrium multiplicity, which implies that the particular signal S is endogenous, i.e., depends on the $\psi^{*}$ chosen. Angeletos and Werning (2006), p.1730, provide a brief discussion and further references regarding this fundamental problem.
} 


\section{Conclusion}

We have introduced heterogenous priors into the canonic global games model of Morris and Shin (2000, 2004), Metz (2002), and Hellwig (2002). The analysis of the baseline model indicates that heterogeneity in priors, unlike heterogeneity in private signals, makes it more difficult for agents to coordinate on multiple equilibria. That is, the origins of belief heterogeneity are of crucial importance to the global games approach: heterogeneity in beliefs, which originates from the variance $\sigma_{\mu}$ in prior expectations, contributes to equilibrium uniqueness. Dispersion in private signals, on the contrary, induces equilibrium multiplicity. In general, the prior's dispersion can ensure unique equilibria as it "thins-out" the group of agents who hold "moderate" beliefs. That is, it reduces the mass of agents with moderate beliefs, and it is this group which can potentially coordinate on multiple equilibria. Equivalently, our results indicate that if player's disagreement, as measured by $\sigma_{\mu}$, is substantial, then they play unique equilibria.

More precisely, we find that if prior dispersion is small, $\left(\sigma_{\mu}<\frac{1}{\sqrt{2 \pi}}\right)$, equilibrium multiplicity depends on the relative precisions of private information $\left(\alpha_{x}\right)$ and the subjective prior $\left(\alpha_{p}\right)$. If priors are sufficiently dispersed, $\left(\sigma_{\mu}>\frac{1}{\sqrt{2 \pi}}\right)$, equilibria are unique irrespective of the relative weights that players assign to private signals and priors. If prior dispersion $\left(\sigma_{\mu}\right)$ vanishes, the original uniqueness condition for the common prior economy $\left(\frac{\sqrt{\alpha_{x}}}{\alpha_{p}} \geq \frac{1}{\sqrt{2 \pi}}\right)$ obtains.

To compare the implications of prior dispersion and dispersion in private information, we have discussed a modified game in which a financial market aggregates private information into a public price signal. Such a modified environment inverts the original findings of Morris and Shin (2000, 2004), Metz (2002), and Hellwig (2002): increases in private information now induce equilibrium multiplicity instead of uniqueness. The same is not true for the role of prior dispersion, which is robust to such a change in the modelling environment and contributes unambiguously towards equilibrium uniqueness. Put differently, the extended model indicates that prior dispersion, rather than arbitrarily precise private information, anchors unique equilibria reliably.

In general, we found that sufficiently dispersed priors ensure unique equilibria across all three public signal environments. Regarding these public signals, it turned out that their implications in themselves varied significantly from case to case: increases in the public signal's precision ensure multiple threshold equilibria in the first two environments, where signals only contain information on the unknown fundamental. The opposite can be true in the third environment, where public signals allow agents to observe each other's actions. If such signals are of high 
quality, they can enable agents to coordinate on one unique threshold equilibrium.

Unlike previous studies, which have introduced heterogenous priors into the global games framework, we have given explicit conditions in terms of means and variances, which allow to study equilibrium multiplicity for a large economy at and away from the private information limit. That is, the present framework facilitates comparative statics in the information structure itself, which allows to characterize and compare the different implications of belief heterogeneity which originate from priors and private signals, respectively. Moreover, these comparative statics are useful in those applications of the global games framework where it is interesting, or necessary, to study the interaction of private and public information away from the limit where private signals are infinitely precise. 


\section{A Game with Exogenous Public Information}

In this appendix, we derive the uniqueness condition that obtains once we augment our baseline model of Section 2 with a public signal:

$$
Z=\theta+\sigma_{z} \varepsilon, \quad \varepsilon \sim \mathcal{N}(0,1)
$$

This signal allows agents to improve their forecast of the probability with which the aggregate attack overwhelms the status quo. The modified payoff indifference condition therefore reads:

$$
P\left(\theta \leq \theta^{*} \mid x^{*}, \mu, Z\right)=c \quad \Leftrightarrow \quad \Phi\left(\sqrt{\alpha}\left(\theta^{*}-\frac{\alpha_{x}}{\alpha} x^{*}-\frac{\alpha_{p}}{\alpha} \mu-\frac{\alpha_{z}}{\alpha} Z\right)\right)=c ; \quad \alpha=\alpha_{x}+\alpha_{p}+\alpha_{z},(16)
$$

where $\Phi()$ is the cumulative of the standard normal distribution. Again, we define $\psi \equiv \frac{\alpha_{x}}{\alpha} x+\frac{\alpha_{p}}{\alpha} \mu$ and rewrite (16) as:

$$
\Phi\left(\sqrt{\alpha}\left(\theta^{*}-\psi^{*}-\frac{\alpha_{z}}{\alpha} Z\right)\right)=c ; \quad \alpha=\alpha_{x}+\alpha_{p}+\alpha_{z} .
$$

The PIC in (17) locates a critical $\psi^{*}$ such that agents attack if $\psi>\psi^{*}$ and do not attack if $\psi<$ $\psi^{*}$. To calculate the mass of attacking agents, we note that $\psi \mid \theta \sim \mathcal{N}\left(\frac{\alpha_{x}}{\alpha} \theta+\frac{\alpha_{p}}{\alpha} E[\mu],\left(\frac{\alpha^{2}}{\alpha_{x}+\alpha_{p}^{2} \sigma_{\mu}^{2}}\right)^{-1}\right)$. Once we define $\alpha_{\psi} \equiv \frac{\alpha^{2}}{\alpha_{x}+\alpha_{p}^{2} \sigma_{\mu}^{2}}$, the CMC can be written as:

$$
P\left(\psi<\psi^{*} \mid \theta^{*}\right)=\theta^{*} \quad \Leftrightarrow \quad \Phi\left(\sqrt{\alpha_{\psi}}\left(\psi^{*}-\frac{\alpha_{x}}{\alpha} \theta^{*}-\frac{\alpha_{p}}{\alpha} E[\mu]\right)\right)=\theta^{*}
$$

Substitution of (17) into (18) again yields a one-dimensional equation in the threshold level $\theta^{*}$ :

$$
\Phi\left(\sqrt{\alpha_{\psi}}\left(\frac{\alpha_{z}+\alpha_{p}}{\alpha} \theta^{*}-\frac{\alpha_{z}}{\alpha} Z-\frac{\alpha_{p}}{\alpha} E[\mu]-\Phi^{-1}(c) \frac{1}{\sqrt{\alpha}}\right)\right)=\theta^{*} .
$$

Accordingly, equilibria are unique if 16

$$
\sqrt{\alpha_{\psi}} \frac{\alpha_{z}+\alpha_{p}}{\alpha} \frac{1}{\sqrt{2 \pi}} \leqq 1 \Leftrightarrow \sqrt{\left(\frac{\sqrt{\alpha_{x}}}{\alpha_{p}+\alpha_{z}}\right)^{2}+\sigma_{\mu}^{2} \frac{1}{\left(1+\frac{\alpha_{z}}{\alpha_{p}}\right)^{2}}} \geq \frac{1}{\sqrt{2 \pi}}
$$

which is what we needed to show.

\section{B Financial Market and Information Aggregation}

In this appendix, we present a financial market that aggregates and reveals dispersed private information, on the fundamental $\theta$, through the stock price. For the present purpose, it is

\footnotetext{
${ }^{16}$ Recall that $\alpha_{\psi} \equiv \frac{\alpha^{2}}{\alpha_{x}+\alpha_{p}^{2} \sigma_{\mu}^{2}}$
} 
convenient to pick a special case of the linear CARA-normal noise trader equilibrium discussed by Grossman and Stiglitz (1976, 1980), Hellwig (1980), and Angeletos and Werning (2006). Stocks are traded at a market price $P$ and pay an unknown amount $\theta$, which represents the firm's fundamental strength. This market price will, in equilibrium, aggregate dispersed private information and reveal the true fundamental $\theta$ partially. Where the partial revelation is due to aggregate noise-trader activity, $\sigma_{\varepsilon} \varepsilon, \varepsilon \sim \mathcal{N}(0,1)$, on the asset's supply side. To characterize the market price signal, we proceed in three steps. First, we guess that there exists a linear price function, $P=\eta_{1} \theta+\eta_{2} \varepsilon+c$. Regarding $\theta$, this function is informationally equivalent to a signal $Z \equiv \frac{P-c}{\eta_{1}}=\theta+\frac{\eta_{2}}{\eta_{1}} \varepsilon$, which reveals the true fundamental with precision $\alpha_{z}=\frac{\eta_{1}^{2}}{\eta_{2}^{2}}$. Second, given this price function, we characterize individual demands based on the information $x, \mu, Z$, and calculate the market equilibrium. Finally, we determine the ratio $\frac{\eta_{1}^{2}}{\eta_{2}^{2}}$ as $\alpha_{x}^{2} \frac{1}{\gamma^{2} \sigma_{\varepsilon}^{2}}$. That is, price signal $Z$ indeed carries information $\alpha_{z}=\alpha_{x}^{2} \frac{1}{\gamma^{2} \sigma_{\varepsilon}^{2}}$ as claimed in (10) in the main text.

5) Demand Agents choose their optimal demands $k_{i}$ for the asset to maximize expected CARA utility:

$$
\begin{aligned}
k_{i} \quad & =\underset{k_{i}}{\arg \max }\left\{\mathbb{E}\left[-e^{-\gamma(\theta-P) k_{i}} \mid x_{i}, \mu, Z\right]\right\} \\
& =\underset{k_{i}}{\arg \max }\left\{\gamma \mathbb{E}\left[(\theta-P) k_{i} \mid x_{i}, \mu, Z\right]-\frac{\gamma^{2}}{2} \operatorname{Var}\left[(\theta-P) k_{i} \mid x_{i}, \mu, Z\right]\right\} \\
& =\underset{k_{i}}{\arg \max }\left\{\gamma\left(\frac{\alpha_{x}}{\alpha} x_{i}+\frac{\alpha_{p}}{\alpha} \mu+\frac{\alpha_{z}}{\alpha} Z-P\right) k_{i}-\frac{\gamma^{2}}{2} k_{i}^{2} \frac{1}{\alpha}\right\}, \quad \alpha=\alpha_{x}+\alpha_{p}+\alpha_{z}
\end{aligned}
$$

and the individual demand function writes:

$$
k_{i}^{d}=\frac{\frac{\alpha_{x}}{\alpha} x_{i}+\frac{\alpha_{p}}{\alpha} \mu+\frac{\alpha_{z}}{\alpha} Z-P}{\alpha^{-1} \gamma} .
$$

6) Equilibrium Aggregate supply $K^{S}=\sigma_{\varepsilon} \varepsilon$, is unobservable and distorted by noise-trader activity $\varepsilon \sim \mathcal{N}(0,1)$. From (21), we find that aggregate demand $K^{D}$ is:

$$
K^{D}=\int_{[0,1]} \int k_{i}(\mu) \phi(\mu) d \mu d i=\frac{\frac{\alpha_{x}}{\alpha} \theta+\frac{\alpha_{p}}{\alpha} E[\mu]+\frac{\alpha_{z}}{\alpha} Z-P}{\alpha^{-1} \gamma} .
$$

Equilibrium requires that:

$$
K^{D}=K^{S} \quad \Leftrightarrow \quad \frac{\frac{\alpha_{x}}{\alpha} \theta+\frac{\alpha_{p}}{\alpha} E[\mu]+\frac{\alpha_{z}}{\alpha} Z-P}{\alpha^{-1} \gamma}=\sigma_{\varepsilon} \varepsilon
$$

To close the argument, we now resubstitute $Z=\frac{P-c}{\eta_{1}}$ and calculate the ratio $\frac{\eta_{1}}{\eta_{2}}$. First, we solve (23) for $P$ to obtain:

$$
P=\frac{\alpha_{x}}{\alpha-\frac{\alpha_{z}}{\eta_{1}}} \theta-\frac{\gamma \sigma_{\varepsilon}}{\alpha-\frac{\alpha_{z}}{\eta_{1}}} \varepsilon+\frac{\alpha_{p} E[\mu]-\alpha_{z} \frac{c}{\eta_{1}}}{\alpha-\frac{\alpha_{z}}{\eta_{1}}} .
$$


Comparison of (24) with our initial guess, $P=\eta_{1} \theta+\eta_{2} \varepsilon+c$, indicates that $\eta_{1}, \eta_{2}$ must satisfy:

$$
\eta_{1}=\frac{\alpha_{x}}{\alpha-\frac{\alpha_{z}}{\eta_{1}}}, \quad \eta_{2}=-\frac{\gamma \sigma_{\varepsilon}}{\alpha-\frac{\alpha_{z}}{\eta_{1}}} ; \quad \alpha=\alpha_{x}+\alpha_{p}+\alpha_{z} .
$$

We quickly determine $\eta_{1}=\frac{\alpha_{x}+\alpha_{z}}{\alpha}, \eta_{2}=-\frac{\left(\alpha_{x}+\alpha_{z}\right) \sqrt{\alpha_{z}}}{\alpha}$ to calculate $\frac{\eta_{1}}{\eta_{2}}=-\alpha_{x} \frac{1}{\gamma \sigma_{\varepsilon}}$. At the same time, it follows from the definition of $\mathrm{Z}$ that $Z=\frac{P-c}{\eta_{1}}=\theta+\frac{\eta_{2}}{\eta_{1}} \varepsilon$. Hence, agents who observe $P$, and know the model's coefficients, receive a signal $Z=\theta-\alpha_{x}^{-1} \gamma \sigma_{\varepsilon} \varepsilon$, as claimed in (10) in the main text.

\section{Proof of Propositions 4 and 5}

In this appendix, we start by laying out the equations that describe equilibria. In turn, we characterize the possible equilibria described in propositions 4 and 5 in two separate paragraphs.

We recall the model from the main text

$$
\begin{aligned}
& S=\Phi^{-1}(A)+\sigma_{\varepsilon} \varepsilon, \quad \varepsilon \sim \mathcal{N}(0,1) \\
& A \equiv P\left(\psi \leq \psi^{*}(S) \mid \theta\right)=\theta \\
& P\left(\theta \leq \theta^{*} \mid x, \mu, S\right)=c
\end{aligned}
$$

To calculate equilibria, we recall that agents act on $x=\theta+\sigma_{x} \xi$, with $\xi \sim \mathcal{N}(0,1)$ and $\theta \mid \mu \sim \mathcal{N}\left(\mu, \sigma_{p}^{2}\right)$, where the prior $\mu$ is distributed over the population as $\mu \sim \mathcal{N}\left(E[\mu], \sigma_{\mu}\right)$. Moreover, we define $\psi \equiv \frac{\alpha_{x}}{\alpha} x+\frac{\alpha_{p}}{\alpha} \mu$ with $\alpha=\alpha_{x}+\alpha_{p}+\alpha_{z}$. The PIC (28) now writes as:

$$
\Phi\left(\sqrt{\alpha}\left(\theta^{*}-\psi^{*}-\frac{\alpha_{z}}{\alpha} Z\right)\right)=c ; \quad \alpha=\alpha_{x}+\alpha_{p}+\alpha_{z} .
$$

Again, (29) defines a critical $\psi^{*}(Z)$ such that agents attack if $\psi \leq \psi^{*}$ and do not attack if $\psi>$ $\psi^{*}$. To calculate the mass of attacking agents, we note that $\psi \mid \theta \sim \mathcal{N}\left(\frac{\alpha_{x}}{\alpha} \theta+\frac{\alpha_{p}}{\alpha} E[\mu],\left(\frac{\alpha^{2}}{\alpha_{x}+\alpha_{p}^{2} \sigma_{\mu}^{2}}\right)^{-1}\right)$. Once we define $\alpha_{\psi} \equiv \frac{\alpha^{2}}{\alpha_{x}+\alpha_{p}^{2} \sigma_{\mu}^{2}}$, the CMC (27) can be written as:

$$
A=P\left(\psi<\psi^{*} \mid \theta^{*}\right)=\theta^{*} \quad \Leftrightarrow \quad \Phi\left(\sqrt{\alpha_{\psi}}\left(\psi^{*}-\frac{\alpha_{x}}{\alpha} \theta^{*}-\frac{\alpha_{p}}{\alpha} E[\mu]\right)\right)=\theta^{*} .
$$

Using this expression for the aggregate attack $A$ in condition (30), we can return to the public signal $S$ in (26) and write:

$$
S=\sqrt{\alpha_{\psi}}\left(\psi^{*}-\frac{\alpha_{x}}{\alpha} \theta-\frac{\alpha_{p}}{\alpha} E[\mu]\right)+\sigma_{\varepsilon} \varepsilon
$$


Where $S$ in (31) is informationally equivalent to a signal

$$
Z(S) \equiv \frac{\alpha}{\alpha_{x}} \psi^{*}(S)-\frac{\alpha}{\alpha_{x}} \frac{1}{\sqrt{\alpha_{\psi}}} S=\theta+\frac{\alpha_{p}}{\alpha_{x}} E[\mu]-\sigma_{\varepsilon} \frac{\alpha}{\alpha_{x}} \frac{1}{\sqrt{\alpha_{\psi}}} \varepsilon .
$$

Regarding (32), we note that $Z(S)$ contains two aspects (i) $Z$ is a noisy public signal which reveals the true state of the economy $\theta$ with precision $\alpha_{z}=\frac{\alpha_{x}^{2} \alpha_{\psi}}{\sigma_{\varepsilon}^{2} \alpha^{2}}=\frac{\alpha_{x}^{2}}{\sigma_{\varepsilon}^{2}\left(\alpha_{x}+\alpha_{p}^{2} \sigma_{\mu}^{2}\right)}$ and (ii) the signal $S$ allows agents to align their strategies $\psi^{*}(S)$. That is, for every given $\bar{Z}$, there may be several $S$ such that $Z(S)=\bar{Z}$. That is, there is a potential source of equilibrium multiplicity, concerning $S$, to which we turn in Paragraph 2) of this appendix. For now, we take $S$ as given and study the threshold equilibria $\theta^{*}(S), \psi^{*}(S)$.

7) Proof of Proposition 4: Multiplicity in Thresholds $\theta^{*}$ For every given signal $S$, we rewrite (30) as:

$$
\psi^{*}=\Phi^{-1}\left(\theta^{*}\right) \frac{1}{\sqrt{\alpha_{\psi}}}+\frac{\alpha_{x}}{\alpha} \theta^{*}+\frac{\alpha_{p}}{\alpha} E[\mu] .
$$

To obtain an equation in $\theta^{*}$ only, we substitute $Z(S)=\frac{\alpha}{\alpha_{x}} \psi^{*}(S)-\frac{\alpha}{\alpha_{x}} \frac{1}{\sqrt{\alpha_{\psi}}} S$ and (33) into (29)). Rearranging then yields:

$$
\Phi\left(\sqrt{\alpha}\left(\frac{\alpha_{p}}{\alpha} \theta^{*}-\frac{1}{\sqrt{\alpha_{\psi}}} \frac{\alpha_{x}+\alpha_{z}}{\alpha_{x}} \Phi^{-1}\left(\theta^{*}\right)-\frac{\left(\alpha_{x}+\alpha_{z}\right) \alpha_{p}}{\alpha_{x} \alpha} E[\mu]+\frac{\alpha_{z}}{\alpha_{x}} \frac{1}{\sqrt{\alpha_{\psi}}} S\right)\right)=c
$$

To derive the uniqueness condition, which ensures that there exist only one $\theta^{*}(S)$ for every given signal $S$, we differentiate (34) with respect to $\theta^{*} \cdot 17$

$$
\phi\left(\Phi\left(\theta^{*}\right)^{-1}\right) \leq \frac{\left(\alpha_{x}+\alpha_{z}\right)}{\alpha_{x}} \sqrt{\left(\frac{\sqrt{\alpha_{x}}}{\alpha_{p}}\right)^{2}+\sigma_{\mu}^{2}},
$$

and hence, threshold equilibria are always unique iff $\frac{1}{\sqrt{2 \pi}} \leq \frac{\left(\alpha_{x}+\alpha_{z}\right)}{\alpha_{x}} \sqrt{\left(\frac{\sqrt{\alpha_{x}}}{\alpha_{p}}\right)^{2}+\sigma_{\mu}^{2}}$. Otherwise, if $\frac{1}{\sqrt{2 \pi}} \geq \frac{\left(\alpha_{x}+\alpha_{z}\right)}{\alpha_{x}} \sqrt{\left(\frac{\sqrt{\alpha_{x}}}{\alpha_{p}}\right)^{2}+\sigma_{\mu}^{2}}$, there may exist up to three threshold equilibria $\theta_{1}^{*}(S), \psi_{1}^{*}(S) ; \theta_{2}^{*}(S), \psi_{2}^{*}(S) ; \theta_{3}^{*}(S), \psi_{3}^{*}(S)$ for every given signal value $S$.

8) Proof of Proposition 5: Multiplicity in Strategies $\psi^{*}(S)$ To preclude multiple solutions $18(\bar{Z})$ to the equation $Z(S)=\bar{Z}$, where $Z(S)=\frac{\alpha}{\alpha_{x}} \psi^{*}(S)-\frac{\alpha}{\alpha_{x}} \frac{1}{\sqrt{\alpha_{\psi}}} S$, it will suffice

\footnotetext{
${ }^{17}$ Note that for $y=\Phi^{-1}\left(\theta^{*}\right)$, we have $\frac{d \theta^{*}}{d y}=\phi(y)$ and thus $\frac{d y}{d \theta^{*}}=\frac{1}{\phi(y)}=\frac{1}{\phi\left(\Phi^{-1}\left(\theta^{*}\right)\right)}$.

${ }^{18}$ The existence of at least one solution is ensured. It follows from (33) that $\lim _{S \rightarrow \infty} \frac{\Phi\left(\theta^{*}(S)\right)^{-1}}{S}$ and $\lim _{S \rightarrow-\infty} \frac{\Phi\left(\theta^{*}(S)\right)^{-1}}{S}$ are constants. Rewriting $Z(S)=\frac{\alpha}{\alpha_{x}} \psi^{*}(S)-\frac{\alpha}{\alpha_{x}} \frac{1}{\sqrt{\alpha_{\psi}}} S$ as $Z(S)=\frac{\alpha}{\alpha_{x}} S\left(\frac{\psi^{*}(S)}{S}-\frac{\alpha}{\alpha_{x}} \frac{1}{\sqrt{\alpha_{\psi}}}\right)$ and recalling $\psi\left(\theta^{*}(S)\right)$ as given in (33) one can show that $Z(S)$ varies with $S$ between $\infty$ and $-\infty$.
} 
to show that $\frac{\partial Z(S)}{\partial S} \mid 32=\frac{\alpha}{\alpha_{x}} \frac{\partial \psi^{*}}{\partial S}-\frac{\alpha}{\alpha_{x}} \frac{1}{\sqrt{\alpha_{\psi}}} \leq 0$. To calculate the derivative $\frac{\partial \psi\left(\theta^{*}(S)\right)}{\partial S}=\frac{\partial \psi^{*}}{\partial \theta^{*}} \frac{\partial \theta^{*}}{\partial S}$,

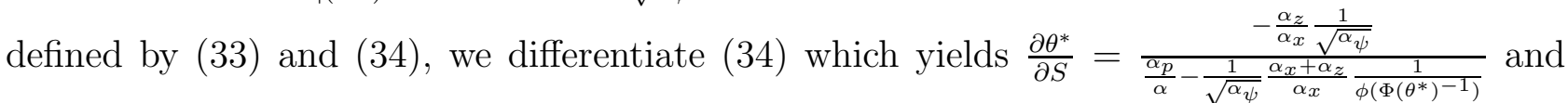
(33), (which is a $1: 1$ mapping between $\psi^{*}$ and $\theta^{*}$ ), to obtain $\frac{\partial \psi^{*}}{\partial \theta^{*}}=\frac{1}{\sqrt{\alpha_{\psi}} \phi\left(\Phi\left(\theta^{*}\right)^{-1}\right)}+\frac{\alpha_{x}}{\alpha}$. Hence, we have

$$
\begin{aligned}
\frac{\partial Z(S)}{\partial S} & =\frac{\alpha}{\alpha_{x}} \frac{\partial \psi^{*}}{\partial S}-\frac{\alpha}{\alpha_{x}} \frac{1}{\sqrt{\alpha_{\psi}}}=\frac{\alpha}{\alpha_{x}} \frac{\partial \psi^{*}}{\partial \theta^{*}} \frac{\partial \theta^{*}}{\partial S}-\frac{\alpha}{\alpha_{x}} \frac{1}{\sqrt{\alpha_{\psi}}} \\
& =\underbrace{-\frac{\alpha}{\alpha_{x}} \frac{1}{\sqrt{\alpha_{\psi}}}}_{-}+\underbrace{\left(\frac{1}{\sqrt{\alpha_{\psi}} \phi\left(\Phi\left(\theta^{*}\right)^{-1}\right)}+\frac{\alpha_{x}}{\alpha}\right)}_{+} \underbrace{\frac{-\frac{\alpha_{z}}{\alpha_{x}} \frac{1}{\sqrt{\alpha_{\psi}}}}{\frac{\alpha_{p}}{\alpha}-\frac{1}{\sqrt{\alpha_{\psi}} \frac{\alpha_{x}+\alpha_{z}}{\alpha_{x}} \frac{1}{\phi\left(\Phi\left(\theta^{*}\right)^{-1}\right)}}} \frac{\alpha}{\alpha_{x}}}_{+/-} .
\end{aligned}
$$

Once we recall that $\alpha_{\psi} \equiv \frac{\alpha^{2}}{\alpha_{x}+\alpha_{p}^{2} \sigma_{\mu}^{2}}$, rearranging (36) gives:

$$
\begin{aligned}
\frac{\partial Z(S)}{\partial S} & =-\frac{\sqrt{\alpha_{x}+\alpha_{p}^{2} \sigma_{\mu}^{2}}}{\alpha_{x}}+\left(\sqrt{\alpha_{x}+\alpha_{p}^{2} \sigma_{\mu}^{2}}+\alpha_{x} \phi\left(\Phi\left(\theta^{*}\right)^{-1}\right)\right) \frac{-\frac{\alpha_{z}}{\alpha_{x}}}{\frac{\phi\left(\Phi\left(\theta^{*}\right)^{-1}\right) \alpha_{p} \alpha_{x}}{\sqrt{\alpha_{x}+\alpha_{p}^{2} \sigma_{\mu}^{2}}}-\left(\alpha_{x}+\alpha_{z}\right)} \\
& =\frac{1}{\alpha_{x}}\left[-\sqrt{\alpha_{x}+\alpha_{p}^{2} \sigma_{\mu}^{2}}+\left(\sqrt{\alpha_{x}+\alpha_{p}^{2} \sigma_{\mu}^{2}}+\alpha_{x} \phi\left(\Phi\left(\theta^{*}\right)^{-1}\right)\right) \frac{-\alpha_{z}}{\frac{\phi\left(\Phi\left(\theta^{*}\right)^{-1}\right) \alpha_{p} \alpha_{x}}{\sqrt{\alpha_{x}+\alpha_{p}^{2} \sigma_{\mu}^{2}}}-\left(\alpha_{x}+\alpha_{z}\right)}\right] \\
& =\frac{1}{\alpha_{x}}\left[-\alpha_{x}\left(\alpha_{p}+\alpha_{z}\right) \phi\left(\Phi\left(\theta^{*}\right)^{-1}\right)+\alpha_{x} \sqrt{\alpha_{x}+\alpha_{p}^{2} \sigma_{\mu}^{2}} \frac{1}{\frac{\phi\left(\Phi\left(\theta^{*}\right)^{-1}\right) \alpha_{p} \alpha_{x}}{\sqrt{\alpha_{x}+\alpha_{p}^{2} \sigma_{\mu}^{2}}}-\left(\alpha_{x}+\alpha_{z}\right)}\right. \\
& =\left[-\left(\alpha_{p}+\alpha_{z}\right) \phi\left(\Phi\left(\theta^{*}\right)^{-1}\right)+\sqrt{\alpha_{x}+\alpha_{p}^{2} \sigma_{\mu}^{2}}\right] \frac{1}{\frac{\phi\left(\Phi\left(\theta^{*}\right)^{-1}\right) \alpha_{p} \alpha_{x}}{\sqrt{\alpha_{x}+\alpha_{p}^{2} \sigma_{\mu}^{2}}}-\left(\alpha_{x}+\alpha_{z}\right)}
\end{aligned}
$$

From (37), and the fact that $\theta^{*} \in(0,1)$, it follows that equilibria in strategies are unique if

$$
\frac{1}{\sqrt{2 \pi}} \leq \sqrt{\left(\frac{\sqrt{\alpha_{x}}}{\alpha_{p}+\alpha_{z}}\right)^{2}+\frac{\sigma_{\mu}^{2}}{1+\frac{\alpha_{z}}{\alpha_{p}}}}, \quad \alpha_{z}=\frac{\alpha_{x}^{2}}{\sigma_{\varepsilon}^{2}\left(\alpha_{x}+\alpha_{p}^{2} \sigma_{\mu}^{2}\right)}
$$

and

$$
\frac{1}{\sqrt{2 \pi}} \leq \sqrt{\frac{\left(\alpha_{x}+\alpha_{z}\right)^{2}}{\alpha_{x} \alpha_{p}^{2}}+\frac{\sigma_{\mu}^{2}\left(\alpha_{x}+\alpha_{z}\right)^{2}}{\alpha_{x}^{2}}}
$$

That is, once (38) and (39) hold, we have $\frac{\partial Z(S)}{\partial S}<0$, which ensures unique solutions $S(\bar{Z})$ to the equation $Z(S)=\bar{Z}$. Comparison shows that inequality (39) is less restrictive than (38) 19 Evaluation of (38) therefore yields:

1. In the limit where $\sigma_{\mu} \rightarrow \infty$, equilibria in strategies are unique.

\footnotetext{
$19 \sqrt{\frac{\alpha_{x}}{\left(\alpha_{p}+\alpha_{z}\right)^{2}}+\frac{\sigma_{\mu}^{2}}{1+\frac{\alpha_{z}}{\alpha_{p}}}} \leq \sqrt{\frac{\left(\alpha_{x}+\alpha_{z}\right)^{2}}{\alpha_{x} \alpha_{p}^{2}}+\frac{\sigma_{\mu}^{2}\left(\alpha_{x}+\alpha_{z}\right)^{2}}{\alpha_{x}^{2}}}$ follows from the inequalities $\frac{\alpha_{x}}{\left(\alpha_{p}+\alpha_{z}\right)^{2}} \leq \frac{\left(\alpha_{x}+\alpha_{z}\right)^{2}}{\alpha_{x} \alpha_{p}^{2}}$ and $\frac{\sigma_{\mu}^{2}}{1+\frac{\alpha_{z}}{\alpha_{p}}} \leq \frac{\sigma_{\mu}^{2}\left(\alpha_{x}+\alpha_{z}\right)^{2}}{\alpha_{x}^{2}}$, which are easy to verify.
} 
2. In the limit 20 where $\alpha_{z} \rightarrow \infty$, there exist multiple equilibria in strategies.

3. Multiple equilibria are ensured in the limit where $\alpha_{x} \rightarrow \infty$.

4. Finally, in the special case where $\sigma_{\mu}^{2}=0$ and $\alpha_{p}=0$, condition (38) collapses into the uniqueness condition $\sqrt{2 \pi} \leq \frac{\sqrt{\alpha_{x}}}{\alpha_{z}}$, of Angeletos and Werning (2006) pp. 1733-1734, which is nested in the present framework.

\section{Alternative Derivation of (17)}

This appendix contains a derivation of (7) which "explicitly" accounts for the influence which the prior's distribution has on the critical mass condition. Recalling the PIC, we have:

$$
P\left(\theta \leq \theta^{*} \mid x^{*}, \mu\right)=c \quad \Leftrightarrow \quad \Phi\left(\sqrt{\alpha}\left(\theta^{*}-\frac{\alpha_{x}}{\alpha} x^{*}-\frac{\alpha_{p}}{\alpha} \mu\right)\right)=c ; \quad \alpha=\alpha_{x}+\alpha_{p}
$$

Regarding the CMC, we now account explicitly for the prior's distribution and write:

$$
A\left(x^{*}(\mu), \theta^{*}\right)=P\left(x \leq x^{*} \mid \theta^{*}\right)=\theta^{*} \quad \Leftrightarrow \quad \int_{-\infty}^{\infty} \Phi\left(\sqrt{\alpha_{x}}\left(x^{*}(\mu)-\theta^{*}\right)\right) \phi(\mu) d \mu=\theta^{*},
$$

where $\Phi()$ represents the cumulative of the standard normal distribution and $\phi(\mu)$ is the normal density of the prior. To show that the pairs $x^{*}(\mu), \theta^{*}$, which solve (40) and (41) are unique if $\sqrt{\left(\frac{\sqrt{\alpha_{x}}}{\alpha_{p}}\right)^{2}+\sigma_{\mu}^{2}} \geq \frac{1}{\sqrt{2 \pi}}$, we solve (40) for the threshold level $x^{*}=-\Phi^{-1}(c) \frac{\sqrt{\alpha}}{\alpha_{x}}+\theta^{*} \frac{\alpha}{\alpha_{x}}-\frac{\alpha_{p}}{\alpha_{x}} \mu$, and substitute $x^{*}$ into (41) to obtain a one-dimensional equation in $\theta^{*}$ alone:

$$
\int_{-\infty}^{\infty} \Phi\left(\sqrt{\alpha_{x}}\left(-\Phi^{-1}(c) \frac{\sqrt{\alpha}}{\alpha_{x}}+\theta^{*} \frac{\alpha}{\alpha_{x}}-\frac{\alpha_{p}}{\alpha_{x}} \mu-\theta^{*}\right)\right) \phi(\mu) d \mu=\theta^{*}
$$

The sufficient condition for uniqueness of the threshold $\theta^{*}$ is therefore:

$$
\frac{\alpha_{p}}{\sqrt{\alpha_{x}}} \frac{1}{\sqrt{2 \pi}} \int_{-\infty}^{\infty} e^{-\left(\sqrt{\alpha_{x}}\left(-\Phi^{-1}(c) \frac{\sqrt{\alpha}}{\alpha_{x}}+\theta^{*} \frac{\alpha_{p}}{\alpha_{x}}-\frac{\alpha_{p}}{\alpha_{x}} \mu\right)\right)^{2} \frac{1}{2}} \phi(\mu) d \mu \leqq 1
$$

To obtain the final condition, we recall that $\mu \sim \mathcal{N}\left(E[\mu], \sigma_{E[\mu]}\right)$, and use the moment generating function for the non-central $\chi^{2}$ distribution in Paragraph 1) to rewrite (42), as:

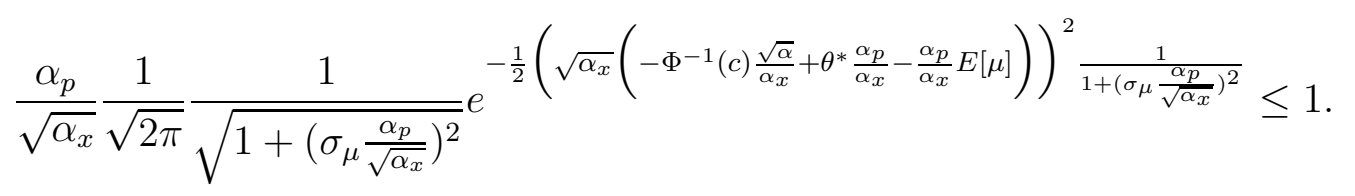

Taking logarithms yields the uniqueness condition $\sqrt{\left(\frac{\sqrt{\alpha_{x}}}{\alpha_{p}}\right)^{2}+\sigma_{\mu}^{2}} \geq \frac{1}{\sqrt{2 \pi}}$.

\footnotetext{
${ }^{20}$ Note that the public signal's precision is endogenous, i.e., given by $\alpha_{z}=\frac{\alpha_{x}^{2}}{\sigma_{\varepsilon}^{2}\left(\alpha_{x}+\alpha_{p}^{2} \sigma_{\mu}^{2}\right)}$. However, the present observation is informative in the sense that the public signal's precision can be varied through $\sigma_{\varepsilon}^{2}$ which is independent of the other parameters.
} 
9) Moment Generating Function Regarding (42), we recall our assumption that $\mu \sim$ $\mathcal{N}\left(E[\mu], \sigma_{E[\mu]}\right)$. We can therefore define $y=-\Phi^{-1}(c) \frac{\sqrt{\alpha}}{\alpha_{x}}+\theta^{*} \frac{\alpha_{p}}{\alpha_{x}}-\frac{\alpha_{p}}{\alpha_{x}} \mu$, where $y \sim \mathcal{N}\left(-\Phi^{-1}(c) \frac{\sqrt{\alpha}}{\alpha_{x}}+\right.$ $\left.\theta^{*} \frac{\alpha_{p}}{\alpha_{x}}-\frac{\alpha_{p}}{\alpha_{x}} E[\mu], \sigma_{\mu} \frac{\alpha_{p}}{\alpha_{x}}\right)$. If a variable $y$ is normally distributed with mean $E[y]$ and variance $\sigma_{y}^{2}$, then $z^{2}=\frac{y^{2}}{\sigma_{y}^{2}}$ is non-centrally $\chi^{2}$ distributed. We can therefore use the moment generating function for the non-central $\chi^{2}$ distribution (Rao (1965), p. 181) 21

$$
E\left[e^{-t z^{2}}\right]=\frac{1}{\sqrt{1+2 t}} e^{\frac{-E[z]^{2} t}{1+2 t}}, \quad t>0
$$

to rewrite (42) as (43). To do so, we set $t=\frac{1}{2} \alpha_{x} \sigma_{y}^{2}=\frac{1}{2} \sigma_{\mu}^{2} \frac{\alpha_{p}^{2}}{\alpha_{x}}$, and substitute $z^{2}=\frac{y^{2}}{\sigma_{y}^{2}}$, with $y=-\Phi^{-1}(c) \frac{\sqrt{\alpha}}{\alpha_{x}}+\theta^{*} \frac{\alpha_{p}}{\alpha_{x}}-\frac{\alpha_{p}}{\alpha_{x}} \mu$, into (42), to obtain.22

$$
\begin{aligned}
& \frac{\alpha_{p}}{\sqrt{\alpha_{x}}} \frac{1}{\sqrt{2 \pi}} \int_{-\infty}^{\infty} e^{\left.-t z^{2} \phi(\mu) d \mu=\mid 44\right)} \frac{\alpha_{p}}{\sqrt{\alpha_{x}}} \frac{1}{\sqrt{2 \pi}} \frac{1}{\sqrt{1+\alpha_{x} \sigma_{y}^{2}}} e^{-\frac{E[y]^{2}}{\sigma_{y}^{2}} \frac{1}{2} \alpha_{x} \sigma_{y}^{2} \frac{1}{1+\alpha_{x} \sigma_{y}^{2}}} \\
& =\frac{\alpha_{p}}{\sqrt{\alpha_{x}}} \frac{1}{\sqrt{2 \pi}} \frac{1}{\sqrt{1+\left(\sigma_{\mu} \frac{\alpha_{p}}{\sqrt{\alpha_{x}}}\right)^{2}}} e^{-\frac{1}{2}\left(\sqrt{\alpha_{x}}\left(-\Phi^{-1}(c) \frac{\sqrt{\alpha}}{\alpha_{x}}+\theta^{*} \frac{\alpha_{p}}{\alpha_{x}}-\frac{\alpha_{p}}{\alpha_{x}} E[\mu]\right)\right)^{2} \frac{1}{1+\left(\sigma_{\mu} \frac{\alpha_{p}}{\left.\sqrt{\alpha_{x}}\right)^{2}}\right.}} .
\end{aligned}
$$

Where the final step from (45) to (46) involves cancelling and resubstitution of $\alpha_{x} \sigma_{y}^{2}=\left(\sigma_{\mu} \frac{\alpha_{p}}{\sqrt{\alpha_{x}}}\right)^{2}$ and $E[y]=-\Phi^{-1}(c) \frac{\sqrt{\alpha}}{\alpha_{x}}+\theta^{*} \frac{\alpha_{p}}{\alpha_{x}}-\frac{\alpha_{p}}{\alpha_{x}} E[\mu]$.

\footnotetext{
${ }^{21} \mathbb{E} e^{t z^{2}} \quad=\quad \frac{1}{\sqrt{2 \pi}} \int_{-\infty}^{\infty} e^{t z^{2}} e^{-\frac{(z-E[z])^{2}}{2}} d z \quad=\quad \frac{1}{\sqrt{2 \pi}} \int_{-\infty}^{\infty} e^{-\frac{\left.(1-2 t) z^{2}-2 E[z] z+E[z]\right]^{2}}{2}} d z=$ $\frac{1}{\sqrt{2 \pi}} \int_{-\infty}^{\infty} e^{-\frac{(\sqrt{1-2 t} z-E[z])^{2}-\frac{E[z]^{2}}{1-2 t}+E[z]^{2}}{2}} d z=\frac{1}{\sqrt{1-2 t} \sqrt{2 \pi}} \int_{-\infty}^{\infty} e^{-\frac{E[z]^{2} t}{1-2 t}} e^{-\frac{(\sqrt{1-2 t} z-E[z])^{2}}{2}} d(\sqrt{1-2 t} z)=\frac{1}{\sqrt{1-2 t}} e^{-\frac{E[z]^{2} t}{1-2 t}}$. ${ }^{22}$ Regarding the equality in (45), we note that $\frac{\alpha_{p}}{\sqrt{\alpha_{x}}} \frac{1}{\sqrt{2 \pi}} \int_{-\infty}^{\infty} e^{-t z^{2}} \phi(\mu) d \mu=$ $\frac{\alpha_{p}}{\sqrt{\alpha_{x}}} \frac{1}{\sqrt{2 \pi}} \int_{-\infty}^{\infty} e^{-t z^{2}} \frac{1}{\sqrt{2 \pi} \sigma_{\mu}} e^{-\frac{(\mu-E[\mu])^{2}}{2 \sigma_{\mu}^{2}}} d \mu \quad=\quad \frac{\alpha_{p}}{\sqrt{\alpha_{x}}} \frac{1}{\sqrt{2 \pi}} \int_{-\infty}^{\infty} e^{-t z^{2}} \frac{1}{\sqrt{2 \pi} \sigma_{\mu}} e^{-\frac{(z-E[z])^{2}}{2}} \sigma_{\mu} d z=$ $\frac{\alpha_{p}}{\sqrt{\alpha_{x}}} \frac{1}{\sqrt{2 \pi}} \int_{-\infty}^{\infty} e^{-t z^{2}} \frac{1}{\sqrt{2 \pi}} e^{-\frac{(z-E[z])^{2}}{2}} d z$. It now follows from the steps in footnote 21, equation (44) respectively, that the equality in (45) holds.
} 


\section{References}

Angeletos, G.-M. and Werning, I. (2006). Crises and prices: Information aggregation, multiplicity, and volatility. American Economic Review, 96(5):1720-1736.

Atkeson, A. (2000). Discussion on morris and shin. In NBER Macroeconomics Annual, pages 161-170. ed. Bernanke, Ben S. and Kenneth Rogoff, Cambridge, MIT Press.

Carlsson, H. and van Damme, E. (1993). Global games and equilibrium selection. Econometrica, 61:989-1018.

Dasgupta, A. (2007). Coordination and delay in global games. Journal of Economic Theory, 134(1):195-225.

Grossman, S. and Stiglitz, J. (1976). Information and competitive price systems. American Economic Review Papers and Proceedings, 66(2):246-253.

Grossman, S. and Stiglitz, J. (1980). On the impossibility of informationally efficient markets. American Economic Review, 70(3):393-408.

Hellwig, C. (2002). Public information, private information, and the multiplicity of equilibria in coordination games. Journal of Economic Theory, 107:191-222.

Hellwig, C., Mukherji, A., and Tsyvinski, A. (2006). Self-fulfilling currency crises: The role of interest rates. American Economic Review, 95(5):1769-1787.

Hellwig, M. (1980). On the aggregation of information in competitive markets. Journal of Economic Theory, 22:477-498.

Izmalkov, S. and Yildiz, M. (2010). Investor sentiments. American Economic Journal: Microeconomics, 2(1):21-38.

Mathevet, L. (2012). Beliefs and rationalizability in games with complementarities. Workingpaper, pages 1-36.

Metz, C. (2002). Public and private information in self-fulfilling currency crises. Journal of Economics, 76:65-85. 
Minelli, E. and Polemarchakis, H. (2003). Information at equilibrium. Economic Theory, $21(2-3): 573-584$.

Morris, S. (1995). The common prior assumption in economic theory. Economics and Philisophy, 11:227-253.

Morris, S. and Shin, H. S. (1998). Unique equilibrium in a model of self-fullfilling currency attacks. American Economic Review, 88(3):578-597.

Morris, S. and Shin, H. S. (2000). Rethinking multiple equilibria in macroeconomic modeling. In NBER Macroeconomics Annual, pages 139-161. ed. Bernanke, Ben S. and Kenneth Rogoff, Cambridge, MIT Press.

Morris, S. and Shin, H. S. (2004). Coordination risk and the price of debt. European Economic Review, 48(1):133-153.

Raiffa, H. and Schlaifer, R. (2000). Applied Statistical Decision Theory. Wiley Classics Library.

Rao, C. R. (1965). Linear Statistical Inference and its Applications. Wiley, New York.

Rubinstein, A. (1989). The electronic mail game: Strategic behaviour under 'almost common knowledge'. American Economic Review, 79(3):385-391.

Sethi, R. and Yildiz, M. (2012). Public disagreement. American Economic Journal: Microeconomics, 4(3):57-95.

Steiner, J. and Stewart, C. (2008). Contagion through learning. Theoretical Economics, 3:431458. 